\title{
Knowledge, Attitudes, and Perceptions about Antibiotic Stewardship Programs among Neonatology Trainees
}

\author{
Ibukunoluwa C. Kalu, MD ${ }^{1}$ Sagori Mukhopadhyay, MD, MMSc ${ }^{2}$ Dmitry Dukhovny, MD, MPH ${ }^{3}$ \\ Rebecca Young, MS $^{1}$ Judith A. Guzman-Cottrill, $\mathrm{DO}^{4}$
}

\footnotetext{
${ }^{1}$ Division of Pediatric Infectious Disease, Department of Pediatrics, Duke University Medical Center, Durham, North Carolina

2 Division of Neonatology, Department of Pediatrics, Children's Hospital of Philadelphia, Perelman School of Medicine, University of

Pennsylvania, Philadelphia, Pennsylvania

${ }^{3}$ Division of Neonatology, Department of Pediatrics, Oregon Health \&

Science University, Portland, Oregon

${ }^{4}$ Division of Pediatric Infectious Disease, Department of Pediatrics, Oregon Health \& Science University, Portland, Oregon
}

Address for correspondence Ibukunoluwa C. Kalu, MD, Department of Pediatrics, Duke University Medical Center, 2301 Erwin Road, DUMC 3499, Durham, NC 27710 (e-mail: ica5@duke.edu).

Am J Perinatol

\begin{abstract}
Keywords

- antibiotic resistance

- neonates

- curriculum development

Objective Antibiotic stewardship should be an essential component of neonatology training as neonatal intensive care units (NICU) have unique stewardship needs. Our aim was to assess neonatology fellowship trainees' knowledge, attitudes, and perceptions about antibiotic stewardship to inform sustainable curriculum development.

Study Design We distributed an electronic survey to neonatology fellows in the United States over 4 months (January-April 2018) via Accreditation Council for Graduate Medical Education program directors.

Results Of 99 programs in the United States with an estimated 700 fellows, 159 (23\%) fellows from 52 training programs (53\%) responded to the survey and 139 (87\%) provided analyzed responses. Majority of respondents were training in southern (59; $42 \%$ ) and northeastern $(43 ; 31 \%$ ) regions and were equally spread across all 3 years of training. One hundred $(72 \%)$ respondents reported an antibiotic stewardship program (ASP) in their institution. While $86 \%$ (120/139) were able to identify the components of an ASP, 59\% (82/139) either did not or were unsure if they had received antibiotic stewardship training during fellowship.

Furthermore, while answering case studies, 124 (89\%) respondents identified the optimal antibiotic for methicillin susceptible Staphylococcus aureus (MSSA) infection and $69(50 \%)$ respondents chose appropriate empiric antibiotics for neonatal meningitis. Notably, fellowship training year was not significantly related to the proportion of incorrect knowledge responses $(p=0.40)$. Most survey respondents $(81 ; 59 \%)$ identified small group sessions as the most useful teaching format, while others chose audit and feedback of individual prescribing behavior (52; 38\%) and didactic lectures (52; $38 \%)$. Finally, ninety-five (69\%) respondents preferred trainee-led ASP interventions targeting focal areas such as antifungal and surgical prophylaxis.
\end{abstract}

received

December 31, 2020

accepted after revision

June 1, 2021 (c) 2021. Thieme. All rights reserved.

Thieme Medical Publishers, Inc.,

333 Seventh Avenue, 18th Floor,

New York, NY 10001, USA
DOI https://doi.org/

10.1055/s-0041-1732418.

ISSN 0735-1631. 
Conclusion Antibiotic stewardship is a critical part of neonatology training. Neonatology fellows report variation in access to ASP during their training. Fellows prefer dedicated trainee-led interventions and stewardship curriculum taught within small group settings to promote targeted NICU ASP.

\section{Key Points}

- Most neonatology programs expose trainees to internal or external antibiotic stewardship programs.

- Over half of fellow trainees are unsure about receiving targeted antibiotic stewardship training.

- Most neonatology fellows prefer a trainee-led antibiotic stewardship intervention.

Antibiotic stewardship is increasingly important in medical training. ${ }^{1}$ The cornerstone of proper antibiotic stewardship centers on educating trainees, who can learn methods for improving antibiotic use to determine future practice patterns. ${ }^{2}$ Despite minimal prior antimicrobial prescribing experience, medical trainees primarily prescribe antibiotics in academic health care settings. ${ }^{3}$ Often, in postgraduate training, antibiotic stewardship teaching occurs in unstructured informal settings with no specific educational tool or measured competencies. ${ }^{4}$ While social media platforms have some success in antibiotic stewardship awareness and education, specific impact on appropriate antibiotic prescribing or uptake by medical trainees are still lacking. ${ }^{1,5}$

Neonatal intensive care units, common in academic centers, are high-risk settings for the emergence and spread of drug-resistant bacteria. Recent studies describe successful NICU stewardship programs led to significant declines in antibiotic use without negative consequences (e.g., late onset sepsis or bacteremia). ${ }^{6}$ Yet, neonatal units are excluded from stewardship interventions particularly when embedded within larger academic hospitals focused on adult care. To resolve this, some NICUs without access to locally tailored interventions participate in national or state-level collaboratives and report significant reductions in antibiotic use. ${ }^{7}$ However, regional differences persist in antibiotic prescribing and stewardship. ${ }^{8}$ Providing neonatology trainees with early exposures to appropriate prescribing practices and the multidisciplinary approach to stewardship will likely carry over into their future clinical practices. Therefore, assessing neonatology fellowship trainees' knowledge and perceptions will highlight deficits in antibiotic stewardship education and inform future curriculum development.

\section{Materials and Methods}

Over a 4-month period (January-April 2018), we performed a 13-item survey study, which was distributed electronically (via unique email access links) by Accreditation Council for Graduate Medical Education program directors to neonatology fellows in the United States. Survey questions are available in Supplementary Material 1 (available in the online version). Survey reminder emails were periodically sent to partial or nonrespondents until the end of the study period. There was no compensation provided to respondents. The objective was to assess neonatology fellows' knowledge, attitudes, and perceptions to inform sustainable NICUspecific antibiotic stewardship curricula. Our secondary aim was to describe regional trends in antibiotic stewardship knowledge, attitudes, and perceptions among neonatology trainees. A multidisciplinary group of neonatologists, pediatric infectious disease specialists and public health officials created survey questions focused on core elements of antibiotic stewardship programs (ASP). Three NICU fellows at different training levels tested questions prior to finalizing the survey tool. Based on their feedback, we adjusted the order of questions and emphasized brevity of survey within the introductory email. Survey response data were summarized in frequency tables. After stratifying respondents according to their NICU's geographical locations, we repeated the data analysis. All available survey responses were included in the analysis regardless of survey completion. Analysis of survey responses was conducted in SAS version 9.4 and Stata 15 by using Chi-squared test or Fisher's exact test for categorical responses, with a significance level of $<0.05$.

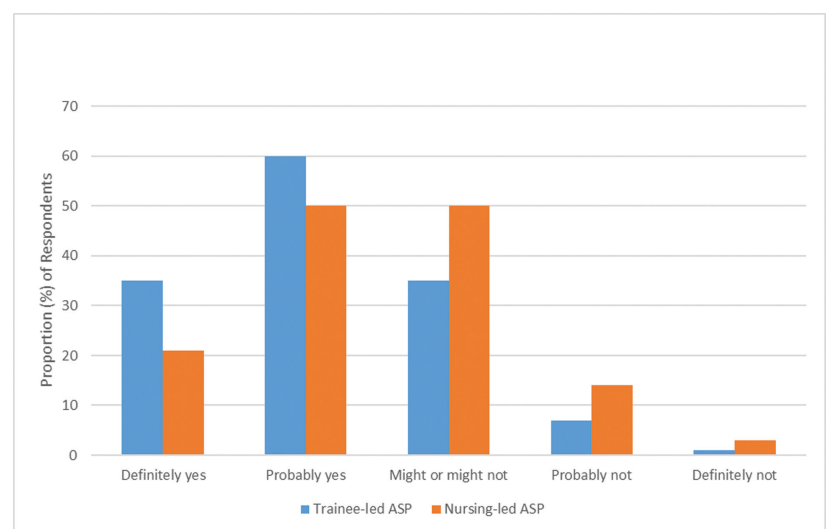

Fig. 1 Stated preferences for leadership of neonatal intensive care unit-antibiotic stewardship program interventions. 


\section{Results}

Using information from the 2017 National Resident Matching Program, we estimated our results for a denominator of 99 NICU fellowship programs and 700 neonatology trainees in fellowship. However, 159 individuals (23\%) from 52 U.S. neonatology-training programs (53\%) attempted the survey, but only 139 (87\%) provided responses to survey questions and data for analysis. One respondent had demographic data but incomplete data for some other sections. Of the 138 completed surveys, respondents represented a wide range of U.S. geographical regions and were equally spread across all 3 years of training such that 48 (35\%) were within their first year of fellowship, 40 (29\%) were within their second year, and 51 (37\%) were within 3 or more years of training (-Supplementary Table 11 [available in the online version]).

Table 1 Neonatology fellows' survey responses assessing Antibiotic Stewardship Program knowledge questions

ASP knowledge Survey questions

Which of the following is/are important components of antibiotic stewardship? Select all that apply.

You are evaluating a premature infant, born at 27 WGA for maternal complications, who was started on gentamicin and ampicillin at birth. Blood cultures are negative at $48 \mathrm{~h}$ and the infant is receiving age appropriate care in the NICU. What is the best course of action?

A 5-day-old full term infant is admitted from the emergency department with hypothermia, and decreased feeding and emesis. Maternal history is notable for inadequate penicillin prophylaxis for Group B Streptococcus colonization. You are concerned for meningitis. What do you do next?

A 42-day-old ex 25 WGA infant developed labile blood pressures, and increased apneic events and respiratory distress $2 \mathrm{~d}$ ago. He has a central catheter and was started on vancomycin and cefotaxime for empiric antimicrobial coverage. His blood cultures are growing methicillin susceptible Staphylococcus aureus? What do you do about his antimicrobial coverage?

\section{Options for survey responses}

Implementing evidence-based recommendations to determine appropriate antibiotic use

Using antibiotics only when it truly indicated

Using the right antibiotic at the right dose

Using the correct route (oral vs. intravenous)

Using antibiotics for the proper duration based on the infectious process

All of the above (correct)

Switch to cefotaxime and continue antibiotics for $7 \mathrm{~d}$

Discontinue antibiotics

Repeat blood cultures to determine duration of antibiotics

Add fluconazole at treatment doses (not for prophylaxis)

Continue ampicillin and gentamicin for $7 \mathrm{~d}$

Initiate vancomycin and a third-generation cephalosporin

Initiate piperacillin/tazobactam and metronidazole

Obtain blood, urine, and CSF cultures and then start ampicillin and a thirdgeneration cephalosporin (correct)

Obtain blood, urine, and CSF cultures and then initiate ampicillin and gentamicin

Start vancomycin and piperacillin/tazobactam

Change to oxacillin

Continue current coverage with vancomycin and cefotaxime

Continue vancomycin and discontinue cefotaxime

Change to ampicillin and gentamicin 1

\begin{tabular}{|c|c|c|c|}
\hline $\begin{array}{l}\text { First-year } \\
\text { fellow }\end{array}$ & $\begin{array}{l}\text { Second-year } \\
\text { fellow }\end{array}$ & $\begin{array}{l}\geq \text { Third-year } \\
\text { fellow }\end{array}$ & Total \\
\hline 18 & 20 & 24 & 62 \\
\hline 16 & 20 & 23 & 59 \\
\hline 18 & 16 & 21 & 55 \\
\hline 13 & 15 & 17 & 45 \\
\hline 17 & 19 & 24 & 60 \\
\hline 42 & 34 & 42 & 118 \\
\hline 0 & 0 & 0 & 0 \\
\hline 47 & 40 & 51 & 138 \\
\hline 0 & 0 & 0 & 0 \\
\hline 0 & 0 & 0 & 0 \\
\hline 0 & 0 & 0 & 0 \\
\hline 0 & 3 & 6 & 9 \\
\hline 0 & 0 & 0 & 0 \\
\hline 25 & 16 & 27 & 68 \\
\hline 22 & 21 & 17 & 60 \\
\hline 0 & 0 & 1 & 1 \\
\hline 42 & 37 & 43 & 122 \\
\hline 0 & 0 & 0 & 0 \\
\hline 4 & 3 & 8 & 15 \\
\hline 1 & 0 & 0 & 1 \\
\hline
\end{tabular}

Abbreviations: CSF, cerebrospinal fluid; NICU, neonatal intensive care unit; WGA, weeks of gestation age. 
Notably, 100 of 138 individuals (72\%) reported an ASP in their institution and most reported an external ASP collaborative. While 86\% (120/139) were able to identify the components of ASP, 59\% (82/139) either did not or were unsure if they had received antibiotic stewardship training during fellowship.

Antibiotic stewardship knowledge questions focused on appropriateness of antibiotics for specific clinical conditions. A total of 124 trainees (89\%) identified optimal MSSA treatment. However, 69 (50\%) neonatology trainees correctly identified appropriate clinical management and choice of empiric antibiotics for neonatal meningitis. Fellowship training year was not significantly related to the proportion of incorrect knowledge responses $(p=0.40)$ ( - Table 1 ).

There was no difference in antibiotic stewardship knowledge by geographic area when comparing the percentage of respondents who correctly identified the appropriate antibiotic choice for neonatal meningitis ( $50 \% ; p=0.59)$ or identified the optimal MSSA treatment $(89 \% ; p=0.30)$. However, significantly fewer respondents in the Southern United States (59\%) reported having an ASP in their NICU, compared with Midwest (87\%), Northeast (81\%) and West (79\%; $p=0.02$ ) United States. There were no differences in the percentage of respondents reportedly receiving antibiotic stewardship education as part of their fellowship (40\%; $p=0.96$ ) or able to correctly identify the components of an ASP by geographic region $(86 \% ; p=0.26)$.

Overall, when all respondents were asked about the most useful format for ASP, 81 fellows (59\%) responded favorably to small group sessions, 52 (38\%) responded favorably to audits, 52 (38\%) to didactic, and 46 (33\%) to online learning modules. Additionally, ninety-five (69\%) trainees would "probably" or "definitely" be interested in a trainee-led ASP (-Fig. 1). Suggested focus areas for a dedicated NICU ASP were necrotizing enterocolitis, antifungal prophylaxis, and appropriate surgical prophylaxis (-Supplementary Table S1 [available in the online version]). Additional survey responses are included in - Supplementary Table S2 (available in the online version).

\section{Discussion}

With limited diagnostic modalities, high rates of antibiotic prescriptions and a neonatal population with nonspecific clinical symptoms attributed to sepsis; antibiotic stewardship education is essential for neonatology trainees. ${ }^{9}$ This survey showed that while most neonatology trainees have an ASP within their institutions and can correctly identify the core ASP components, more than half of respondents were unable to report specific antibiotic stewardship training during fellowship. The lack of targeted ASP training might explain the differences in antibiotic stewardship knowledge and attitudes across trainee levels and geographical areas. Since medical training occurs predominantly via didactic lectures, it was enlightening to see our survey results support the use of small group sessions for ASP education.

Implementation of targeted NICU ASP interventions and appropriate use of antibiotics should decrease deleterious impact of excess antibiotic use while maximizing benefits from optimized use. ${ }^{10,11}$ Neonatology fellows' responses showed preference for a trainee-led NICU ASP suggesting stewardship interventions targeting trainee-prescribing behavior might be more impactful if championed by trainees. Practically, most ASP programs will require infectious disease, neonatology, or pharmacy faculty leadership to provide initial training for fellows but targeted NICU ASP interventions may succeed with fellow leadership or championing. Additionally, survey respondents identified clinical topics without clear antibiotic prescribing guidelines, specifically necrotizing enterocolitis, antifungal prophylaxis, and duration of surgical prophylaxis, as targeted areas for NICU ASP education. These results can inform strategies to improve ASP education and create opportunities for involvement of trainees since most preferred trainee-led ASP. ${ }^{2}$

Despite identifying targeted areas for ASP curriculum for neonatology trainees, the low response rate and unequal responses across geographical regions limit broad application of survey results. Additionally, survey findings cannot inform specific recommendations for individual institutions due to anonymous responses. Yet, it is evident that antibiotic stewardship should remain a critical part of neonatology training. While most institutions have a stewardship program, a dedicated curriculum incorporating small group sessions and didactics may be beneficial for educating neonatology trainees. Additionally, there is an urgent need for future studies assessing knowledge retention and impact on antibiotic prescribing.

Note

This project was previously presented at IDWeek 2019

\section{Conflict of Interest}

None declared.

\section{Acknowledgments}

The authors would like to acknowledge Arjun Srinivasan, MD, and Karen Puopolo, MD, PhD, for editorial feedback on survey questions and support for this research. They also thank the members of SHEA Pediatric Leadership Council for supporting this project.

\section{References}

1 Silverberg SL, Zannella VE, Countryman D, et al. A review of antimicrobial stewardship training in medical education. Int J Med Educ 2017;8:353-374

2 Ohl CA, Luther VP. Health care provider education as a tool to enhance antibiotic stewardship practices. Infect Dis Clin North Am 2014;28(02):177-193

3 Charani E, Cooke J, Holmes A. Antibiotic stewardship programmes-what's missing? J Antimicrob Chemother 2010;65 (11):2275-2277

4 Cummings CL, Geis GM, Kesselheim JC, Sayeed S. Ethics and professionalism education during neonatal-perinatal fellowship training in the United States. J Perinatol 2015;35(10):875-879

5 Pisano J, Pettit N, Bartlett A, et al. Social media as a tool for antimicrobial stewardship. Am J Infect Control 2016;44(11): 1231-1236

6 Nzegwu NI, Rychalsky MR, Nallu LA, et al. Implementation of an antimicrobial stewardship program in a neonatal intensive care unit. Infect Control Hosp Epidemiol 2017;38(10):1137-1143 
7 Dukhovny D, Buus-Frank ME, Edwards EM, et al. A collaborative multicenter QI initiative to improve antibiotic stewardship in newborns. Pediatrics 2019;144(06):e20190589

8 Ho T, Buus-Frank ME, Edwards EM, et al. Adherence of newbornspecific antibiotic stewardship programs to CDC recommendations. Pediatrics 2018;142(06):e20174322

9 Cantey JB, Patel SJ, Flenady V. Antimicrobial stewardship in the NICU. Infect Dis Clin North Am 2014;28(02):247-261
10 Ting JY, Synnes A, Roberts A, et al;Canadian Neonatal Network Investigators. Association between antibiotic use and neonatal mortality and morbidities in very low-birth-weight infants without culture-proven sepsis or necrotizing enterocolitis. JAMA Pediatr 2016;170(12):1181-1187

11 Cantey JB, Pyle AK, Wozniak PS, Hynan LS, Sánchez PJ. Early antibiotic exposure and adverse outcomes in preterm, very low birth weight infants. J Pediatr 2018;203:62-67 